Discrete Comput Geom 32:447-457 (2004)

DOI: $10.1007 / \mathrm{s} 00454-004-1132-4$

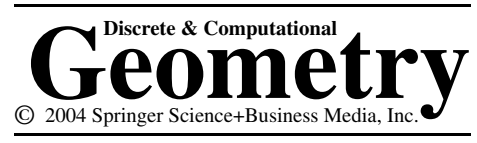

\title{
Isoradial Bodies*
}

\author{
René Brandenberg, ${ }^{1}$ Abhi Dattasharma, ${ }^{2}$ Peter Gritzmann, ${ }^{1}$ and David Larman ${ }^{3}$ \\ ${ }^{1}$ Zentrum Mathematik, Technische Universität München, \\ Boltzmannstr. 3, D-85747 Garching bei München, Germany \\ \{brandenb,gritzman\}@ma.tum.de \\ ${ }^{2}$ Strand Genomics Pvt. Ltd., 237 C V Raman Avenue, \\ Bangalore 560080, India \\ abhi@strandgenomics.com \\ ${ }^{3}$ Department of Mathematics, University College London, \\ London WC1E 6BT, England \\ d.larman@ucl.ac.uk
}

\begin{abstract}
In this paper we show that for any dimension $d \geq 2$ there exists a non-spherical strongly isoradial body, i.e., a non-spherical body of constant breadth, such that its orthogonal projections on any subspace has constant in- and circumradius. Besides the curiosity aspect of these bodies, they are highly relevant for the analysis of geometric inequalities between the radii and their extreme cases.
\end{abstract}

\section{Introduction}

It is about a century since Jung [31] and Steinhagen [39] published their famous articles about the upper bounds on the circumradius-diameter ratio, and the width-inradius ratio in euclidean spaces, respectively. Since then, the four quantities inradius, width, circumradius, and diameter have been studied quite thoroughly [6]-[8], [18]-[20], [33], [36], and [38].

In [24] Gritzmann and Klee consider the two more general classes of inner and outer radii in arbitrary Minkowski spaces. In euclidean spaces the outer $j$-radius $R_{j}(C)$,

* The research of the first author was supported by the European Union, through a Marie Curie Fellowship, Contract-No. HPMT-CT-2000-00037. The research of the second and third authors was supported by the Deutsche Forschungsgemeinschaft, through Grant GR 993/7-1. The research of the fourth author was supported by the Deutsche Forschungsgemeinschaft, through the Graduiertenkolleg "Angewandte Algorithmische Mathematik", TU München. 
$1 \leq j \leq d$, of a convex body $C$ is the minimum of the circumradii of the projections of $C$ on each $j$-dimensional subspace (thereafter called $j$-space). In particular, the outer $d$-radius $R_{d}(C)$ is the usual circumradius of $C$ and $R_{1}(C)$ is half of the width of $C$. Correspondingly, the inner $j$-radius $r_{j}(C)$ of $C$ is defined as the radius of the largest $j$-dimensional ball that fits into $C$. For simplicity of notation we abbreviate $r_{j}(C)$ and $R_{j}(C)$ to $r_{j}$ and $R_{j}$, respectively, whenever there is no risk of confusion. Note that $r_{d}$ is just the usual inradius and $r_{1}$ is half of the diameter. The inner and outer radii are dual to each other in the sense that for a symmetric convex body $C$, i.e., when $C=-C$, and its polar $C^{\circ}, r_{j}(C) R_{j}\left(C^{\circ}\right)=1$. Radii of convex bodies find applications in many areas, e.g., computer graphics, robotics, pattern recognition, non-linear global optimization, sensitivity analysis of linear programming, and computational metrology [1], [25]. For some newer results about radii see [2]-[5], [9], [10], [22], [24], [27], [28], and [34]. Also algorithmic problems of the computation and approximation of radii, and their complexity have been studied in great detail; see [12], [13], and [25], and the literature given there.

In [11] the existence of bodies of constant breadth of any dimension, which have the additional property that each of their (orthogonal) projections on at least two-dimensional subspaces is different from a disk was shown. These bodies were called totally nonspherical. Totally non-spherical bodies fulfill the following inequality chain:

$$
r_{d} \leq \cdots \leq r_{2}<r_{1}=R_{1}<R_{2} \leq \cdots \leq R_{d} .
$$

Especially, showing the existence of convex bodies for which $r_{1}<R_{2}$ significantly improves the results of Eggleston [21] and Weißbach [40], who constructed convex bodies with $r_{1}<R_{d-1}$. Together with some standard observations the existence of the totally non-sphericals proves that Fig. 1 is complete in the following sense: for any two radii which are not connected by a directed path, there exist convex bodies $C_{1}, C_{2}$ such that the relationship between the two radii is "less than" for $C_{1}$ and "greater than" for $C_{2}$. Typical examples for such pairs of convex bodies $C_{1}, C_{2}$ are certain centrally symmetric convex bodies, particularly ellipsoids with axes of mutually different length and simplices or totally non-spherical bodies.

In this paper we drive (1) to the extreme by constructing convex bodies in $\mathbb{E}^{d}$ for which

$$
r_{d}=\cdots=r_{2}<r_{1}=R_{1}<R_{2}=\cdots=R_{d} .
$$

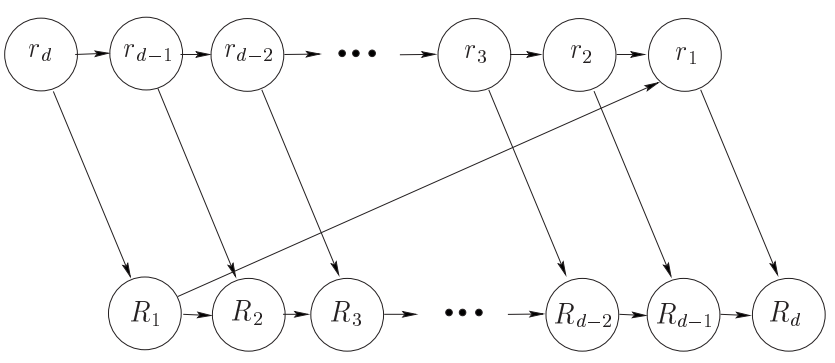

Fig. 1. The arcs imply a less than or equal relationship (from their origin to their sink) between the two corresponding radii, that holds for all $d$-dimensional convex bodies. 
For reasons that will become clear later, convex bodies with

$$
r_{d}=\cdots=r_{2} \leq r_{1}=R_{1} \leq R_{2}=\cdots=R_{d}
$$

are called strongly isoradial. Trivially, the unit ball is strongly isoradial. Hence the main result of this paper is the construction of non-spherical strongly isoradial bodies, which are highly relevant for deriving complete systems of inequalities for radii and their corresponding Blaschke-Santaló diagrams (see [9], [15]-[17] and [35]).

There is another aspect worth mentioning. In [29] Hilbert and Cohn-Vossen asked which properties uniquely characterize the ball in three dimensions? They listed eleven properties, some of which do characterize the ball while others do not. Among the latter is the constant breadth (they use the term constant width) property. A well-known extension of the concept of constant breadth is that of constant outer and inner $j$-measures, $j=$ $1, \ldots, d-1$ [8], [14], [32]. It is known that the three classes, bodies of constant breadth, bodies of constant inner 1-measure, and bodies of constant outer 1-measure, coincide. Firey [23] showed that non-spherical bodies of constant outer $j$-measure exist for all $j \in\{1, \ldots, d-1\}$. On the negative side, it was recently shown that there do not exist non-spherical bodies of constant breadth and constant brightness (constant outer $(d-1)$ measure) in 3-space [30]. Hence the combination of these two properties does indeed characterize the ball. As we will see, for strongly isoradial bodies, each outer and inner $j$-radius is invariant with respect to all subspaces of appropriate dimension. However, as our construction shows, this invariance does not characterize the ball.

This paper is organized as follows: Section 2 contains some preliminaries. Section 3 studies the concept of isoradiality in detail. The construction of strongly isoradial bodies can be found in Section 4 .

\section{Preliminaries}

Let $\mathbb{E}^{d}=\left(\mathbb{R}^{d},\|\cdot\|\right)$ be the $d$-dimensional euclidean space, let $\mathbb{B}^{d}$ and $\mathbb{S}^{d-1}$ be the unit ball and the unit sphere, respectively, and let $\langle\cdot, \cdot\rangle$ denote the usual scalar product $\langle x, y\rangle=x^{T} y$. The linear, positive, and convex hull of some subset $S \subset \mathbb{E}^{d}$ are denoted by $\operatorname{lin}(S), \operatorname{pos}(S)$, and $\operatorname{conv}(S)$, respectively, while for $j \in\{0, \ldots, d\}, \mathcal{L}_{j, d}$ and $\mathcal{A}_{j, d}$ denote the set of all $j$-dimensional linear or affine subspaces of $\mathbb{E}^{d}$, respectively. For $A \subset \mathbb{E}^{d}$ and $F \in \mathcal{L}_{j, d}$, let $A \mid F$ denote the (orthogonal) projection of $A$ on $F$. If $s \in \mathbb{E}^{d} \backslash\{0\}$ and $F=(\operatorname{lin}(\{s\}))^{\perp}$, i.e., $F$ is the hyperplane orthogonal to the line spanned by $s$, we also use the abbreviation $A_{s}$ for $A \mid F$ and refer to this set as the projection of $A$ parallel to $s$. For any two sets $A, B \subset \mathbb{E}^{d}$ the Minkowski sum $A+B$ is defined as $A+B=\left\{a+b \in \mathbb{E}^{d}: a \in A \wedge b \in B\right\}$.

A subset $C$ of $\mathbb{E}^{d}$ is called a convex body if it is bounded, closed, convex, and contains an inner point. Now for any $j \in\{1, \ldots, d\}$ the inner $j$-radius $r_{j}(C)$ of $C$ is the radius of a largest $j$-dimensional ball that fits into $C$, more formally

$$
r_{j}(C)=\max \left\{\rho \geq 0: \exists q \exists F: q \in F \in \mathcal{A}_{j, d} \wedge\left(q+\rho \mathbb{B}^{d}\right) \cap F \subset C\right\} .
$$

The outer $j$-radius $R_{j}(C)$ is defined by

$$
R_{j}(C)=\min \left\{\rho \geq 0: \exists E: E \in \mathcal{A}_{d-j, d} \wedge C \subset E+\rho \mathbb{B}^{d}\right\} .
$$


Since we are restricting ourselves to euclidean spaces, $R_{j}(C)$ coincides with the circumradius of a minimal (with respect to circumradii) projection of $C$ on $j$-spaces. While $r_{1}$ can also be defined in terms of maximal projections this is of course not true for the inner radii in general. As an example, the inner 2-radius $r_{2}$ of the regular 3-simplex with edge-length 1 is $\sqrt{1 / 12}$ while the simplex can be projected onto a plane square whose inradius is $\sqrt{1 / 8}$; see [10]).

A superscript $k$ usually indicates that we consider a functional or object relative to some explicitly given $k$-dimensional affine space $F$. (For example, for $s \in \mathbb{E}^{d} \backslash\{0\}$, $R_{j}^{d-1}\left(C_{s}\right)$ is the outer $j$-radius of the projection $C_{s}$ of $C$ parallel to $s$ while $\mathbb{B}^{k}$ denotes the unit ball in $F$ after identifying $F$ with $\mathbb{E}^{k}$.) For $t \in \mathbb{S}^{d-1}$ the $t$-length of $C$ is defined as $l_{t}(C)=\max \left\{\lambda: \exists c, c^{\prime}: c, c^{\prime} \in C \wedge c^{\prime}=c+\lambda t\right\}$ and the $t$-breadth $b_{t}(C)$ is given by $b_{t}(C)=\max _{c \in C}\langle c, t\rangle-\min _{c \in C}\langle c, t\rangle$. The diameter $\operatorname{diam}(C)$ of $C$ is defined as $\operatorname{diam}(C)=\max _{c_{1}, c_{2} \in C}\|c-t\|$, and width $(C)=\min _{t \in \mathbb{S}^{d-1}} b_{t}(C)$ is the width of $C$. Note that the diameter is the maximum distance between two points within $C$ and the width is the minimum distance of two parallel hyperplanes which support $C$. Clearly, the radii of a convex body are invariant under rigid motions, and, of course, the ratio of two radii is invariant under (non-zero) dilatation. The following propositions are all standard fare; see, e.g., [24].

Proposition 2.1. Let $C$ be a convex body in $\mathbb{E}^{d}$. Then

(a) $\operatorname{diam}(C)=\max _{t \in \mathbb{S}^{d-1}} l_{t}(C)=\max _{t \in \mathbb{S}^{d-1}} b_{t}(C)=2 r_{1}(C)$ and

(b) $\operatorname{width}(C)=\min _{t \in \mathbb{S}^{d-1}} l_{t}(C)=\min _{t \in \mathbb{S}^{d-1}} b_{t}(C)=2 R_{1}(C)$.

Proposition 2.2. Let $C$ be a convex body in $\mathbb{E}^{d}$ and let $j \in\{1, \ldots, d\}$. Then

(a) $R_{1}(C) \leq \cdots \leq R_{d}(C)$,

(b) $r_{1}(C) \geq \cdots \geq r_{d}(C)$,

(c) $R_{1}(C) \leq r_{1}(C)$, and

(d) $r_{j}(C) \leq R_{d+1-j}(C)$.

If the $t$-breadth $b_{t}(C)$ is constant for all $t \in \mathbb{S}^{d-1}$ (so that $\operatorname{diam}(C)=\operatorname{width}(C)$ ), then $C$ is called a body of constant breadth. A convex body $C$ is called complete if $r_{1}(D)>r_{1}(C)$ for all $D \supsetneqq C$. If $C$ is a convex body, then a convex body $\hat{C}$ is called a completion of $C$, if $C \subset \hat{C}, \hat{C}$ is complete and $r_{1}(\hat{C})=r_{1}(C)$.

Proposition 2.3 gives some known facts about bodies of constant breadth [8], [37], which we use later; for surveys see [14], and [26].

Proposition 2.3. Suppose $C \subset \mathbb{E}^{d}$.

(a) The following three statements are equivalent:

(i) $C$ is of constant breadth.

(ii) For each $s \in \mathbb{S}^{d-1}$ the projection $C_{s}$ is a body of constant breadth.

(iii) $C$ is complete.

(b) There exists at least one completion of $C$, which also has the same circumsphere. 
(c) If $C$ is a body of constant breadth, then its incenter (is unique and) coincides with its circumcenter, and $r_{d}(C)+R_{d}(C)=2 r_{1}(C)$.

\section{Isoradiality}

By Proposition 2.2, $r_{d} \leq R_{1} \leq r_{1} \leq R_{d}$. Hence in $\mathbb{E}^{2}$ the radii are linearly ordered. Figure 1 however depicts the fact that in higher dimensions this is not the case. For instance, for symmetric convex bodies $R_{1} \leq r_{d-1}$ and $R_{d-1} \leq r_{1}$ hold, with "=" only in some very special cases; while for the regular simplex $r_{d-1}<R_{1}$, for $d \geq 3$, and $r_{1}<R_{d-1}$, for $d \geq 4$ [2], [40]. Three-dimensional convex bodies with $r_{1}<R_{2}$ were first described in [21]; see also [40], and the existence of totally non-spherical bodies of constant breadth shows that $r_{2}<R_{1}$ and $r_{1}<R_{2}$ is possible in all dimensions [11].

In any case, if $d \geq 3$ there is not even a full description of all relations between any three radii (the so-called Blaschke-Santaló diagrams) yet; see [9]. A particularly important role in the two-dimensional analysis is played by the bodies of constant breadth, see [15]-[17] and [35], for which $r_{1}=R_{1}$. Hence it is natural to generalize this notion and to study classes of convex bodies for which various other radii coincide. As the nonspherical strongly isoradial bodies show, such classes are richer than one might at first expect. By forming outer parallel bodies of such convex bodies it becomes apparent that such inequalities induce non-trivial boundary structures in the corresponding BlaschkeSantaló diagrams; see also [9].

Before constructing strongly isoradial bodies, we give a formal definition of the isoradiality notions that we use, and prove some preliminary results.

Definition 3.1. Let $C$ be a convex body in $\mathbb{E}^{d}$, let $j \in\{1, \ldots, d-1\}$, let $\mathcal{I}=$ $\bigcup_{k=1}^{d-1}\left\{r_{k}, R_{k}\right\}$, and let $\emptyset \neq \mathcal{R} \subset \mathcal{I}$. Then we call $C$

(a) outer $j$-isoradial or $R_{j}$-isoradial if for every $E \in \mathcal{L}_{d-j, d}$ there exists $p \in \mathbb{E}^{d}$ such that $(p+E)+R_{j}(C) \mathbb{B}^{d} \supset C$,

(b) inner $j$-isoradial or $r_{j}$-isoradial if for every $F \in \mathcal{L}_{j, d}$ there exists $q \in \mathbb{E}^{d}$ such that $\left(q+r_{j}(C) \mathbb{B}^{d}\right) \cap(q+F) \subset C$,

(c) $\mathcal{R}$-isoradial if $C$ is $r_{j}$ - and $R_{j}$-isoradial for all $r_{j}, R_{j} \in \mathcal{R}$,

(d) totally isoradial if $C$ is $\mathcal{I}$-isoradial, and

(e) strongly isoradial if

$$
r_{d}(C)=\cdots=r_{2}(C) \leq R_{1}(C)=r_{1}(C) \leq R_{2}(C)=\cdots=R_{d}(C) .
$$

As an example, note that by Proposition 2.3(a) every body of constant breadth is $\left\{r_{1}, R_{1}\right\}$-isoradial and vice versa. Therefore, in $\mathbb{E}^{2}$ the bodies of constant breadth are the only totally isoradial bodies. In higher dimensions the picture is much more complicated.

Also note that a convex body $C$ is $R_{j}$-isoradial if and only if $R_{j}^{k}(C \mid F)$ is independent of $F \in \mathcal{L}_{k, d}$ for each $k \in\{j, \ldots, d\}$. Similarly, $C$ is $r_{j}$-isoradial if and only if for each $k \in$ $\{j, \ldots, d\}$ and $F \in \mathcal{L}_{j, d}$ there exists a point $q \in \mathbb{E}^{d}$ such that $r_{j}^{k}(C \cap(F+q))=r_{j}(C)$.

From the definition it is not immediately clear that any strongly isoradial body is totally isoradial. However, Lemma 3.2 shows that even a somewhat stronger statement holds. 
Lemma 3.2. Let $j \in\{1, \ldots, d-1\}$. Every convex body $C$ with $R_{j}(C)=\cdots=R_{d}(C)$ is $\left\{R_{j}, \ldots, R_{d-1}\right\}$-isoradial, and every convex body $C$ with $r_{d}(C)=\cdots=r_{j}(C)$ is $\left\{r_{j}, \ldots, r_{d-1}\right\}$-isoradial.

Proof. Clearly,

$$
R_{j}(C)=\min \left\{R_{j}(C \mid F): F \in \mathcal{L}_{d-j, d}\right\} \leq \max \left\{R_{j}(C \mid F): F \in \mathcal{L}_{d-j, d}\right\} \leq R_{d}(C) .
$$

Hence isoradiality follows from $R_{j}(C)=R_{d}(C)$. A similar argument holds for the inner radii.

Corollary 3.3. Any strongly isoradial body is totally isoradial.

As an example, an ice cream cone

$$
\operatorname{conv}\left(\left\{(0,0,1)^{\mathrm{T}}\right\} \cup\left(\mathbb{B}^{2} \times\{0\}\right)\right)
$$

satisfies $R_{2}(C)=R_{3}(C)$ and therefore is $R_{2}$-isoradial. Note, however, that the converse of Lemma 3.2 is not true since two-dimensional convex bodies $C$ of constant breadth that are different from $\mathbb{B}^{2}$ fulfill neither $R_{1}(C)=R_{2}(C)$ nor $r_{1}(C)=r_{2}(C)$.

The following lemma is fundamental since it greatly reduces the requirements for the core construction needed to prove the existence of non-spherical strongly isoradial bodies.

Lemma 3.4. Let $C$ be a convex body and let $j \in\{2, \ldots, d-1\}$ such that $r_{1}(C)<$ $R_{j}(C)=R_{d}(C)$. Then there exists a completion $\hat{C}$ of $C$ such that

$$
r_{d}(\hat{C})=r_{j}(\hat{C})<R_{1}(\hat{C})=r_{1}(\hat{C})<R_{j}(\hat{C})=R_{d}(\hat{C}) .
$$

Proof. It follows from Proposition 2.3(b) that there exists a body of constant breadth $\hat{C}$ with $\hat{C} \supset C$ such that

$$
R_{j}(\hat{C}) \leq R_{d}(\hat{C})=R_{d}(C)=R_{j}(C) \leq R_{j}(\hat{C}) .
$$

Thus the relationship holds with equality and every term is greater than $r_{1}(C)=r_{1}(\hat{C})$. Hence, by Proposition 2.3(a),

$$
R_{1}(\hat{C})=r_{1}(\hat{C})<R_{j}(\hat{C})=R_{d}(\hat{C}) .
$$

Now it follows from Proposition 2.3(c) that

$$
r_{d}(\hat{C})+R_{d}(\hat{C})=2 r_{1}(\hat{C}) .
$$

Since for each $F \in \mathcal{L}_{j, d}$ the projection $\hat{C} \mid F$ is also a body of the same constant breadth,

$$
r_{j}(\hat{C} \mid F)+R_{j}(\hat{C} \mid F)=2 r_{1}(\hat{C} \mid F)
$$


holds. By Lemma 3.2,

$$
R_{j}(\hat{C} \mid F)=R_{j}(\hat{C})=R_{d}(\hat{C})
$$

for all $F \in \mathcal{L}_{j, d}$. Hence, we obtain $r_{j}(\hat{C} \mid F)=r_{d}(\hat{C})$ for all $F \in \mathcal{L}_{j, d}$, and therefore $r_{j}(\hat{C} \cap E) \leq r_{d}(\hat{C})$ for all $E \in \mathcal{A}_{j, d}$. This implies that $r_{d}(\hat{C})=r_{j}(\hat{C})$. Since $\hat{C}$ is a non-trivial body of constant breadth, $r_{d}(\hat{C})<R_{1}(\hat{C})$. Thus

$$
r_{d}(\hat{C})=r_{j}(\hat{C})<R_{1}(\hat{C})=r_{1}(\hat{C})<R_{j}(\hat{C})=R_{d}(\hat{C}),
$$

which completes the proof.

\section{Dark Orbits}

In order to produce non-spherical isoradial bodies we adjust a construction in [11] of a packing of spherical caps that is intersected by each great 2-circle. More precisely, we prove the following theorem.

Theorem 4.1. For any dimension $d \geq 3$ there exists a finite set of pairwise disjoint closed spherical caps $\pm C_{1}, \ldots, \pm C_{m}$ on $\mathbb{S}^{d-1}$ such that every great 2 -circle on $\mathbb{S}$ meets the relative interior of at least one pair $\pm C_{i}$.

Any packing of spherical caps according to Theorem 4.1 is called a dark orbit.

The construction proceeds in four steps.

Step 1 . The starting point is the construction of a dark cloud.

Let $H$ be a hyperplane in $\mathbb{E}^{d-1}$, let $\rho>0$, let $B$ be a ball of radius 1 in $H$, and let $\mathcal{P}$ be a packing of $\rho B$ in $H$. Further, let $t \in \mathbb{S}^{d-2}$ be orthogonal to $H$, let $n \in \mathbb{N}$, and let $a_{1}, \ldots, a_{n} \in H$. Then the set

$$
K=\bigcup_{i=1}^{n}\left(a_{i}+i t+\mathcal{P}\right)
$$

is called a dark cloud (for $H$ ) in $\mathbb{E}^{d-1}$ if each line that intersects $H$ in a single point also meets $K$.

We refer to $H$ as the shadowed hyperplane, to $\mathcal{P}$ as the underlying packing and to $\rho$ as the radius of the balls. The existence of dark clouds is long known; see [11] for a specific construction. In particular, the radius of the balls can be assumed to be sufficiently close to 0 so that the distance of the different layers exceeds the radius by any positive factor that one likes. Also, the underlying packing $\mathcal{P}$ can be restricted to lattice packings. By increasing the number of layers the distance of any two balls in $\mathcal{P}$ can also be made as large as necessary for the subsequent construction. Finally, suitable scalings can be applied to change the "absolute metric" of the construction without changing the ratios.

Step 2. Naturally, we cannot only shadow a hyperplane but-with suitable finite unions of dark clouds-also darken the whole unit sphere. In fact, let $\varepsilon \in(0,1)$ and let $A_{\varepsilon}=$ $\left\{x \in \mathbb{E}^{d-1}: 1 \leq\|x\| \leq 1+\varepsilon\right\}$. Further, let $P$ be a simple polytope with

$$
\mathbb{B}^{d-1} \subset P \subset(1+\varepsilon / 3) \mathbb{B}^{d-1},
$$


and let $H_{1}, \ldots, H_{k}$ denote the affine hulls of its facets. Then take the union of $k$ dark clouds $K_{1}, \ldots, K_{k}$ shadowing $H_{1}, \ldots, H_{k}$, respectively, scaled so that for $i=1, \ldots, k$ each dark cloud $K_{i}$ is contained in the strip between $H_{i}$ and $(1+\varepsilon / 6) H_{i}$ and the radius of the balls is less than $\varepsilon / 3$. A simple calculation then shows that $(1+\varepsilon / 6) P \subset$ $(1+2 \varepsilon / 3) \mathbb{B}^{d-1}$. Now, let $L$ be a line that intersects $\mathbb{B}^{d-1}$. Then $L$ also meets $(1+\varepsilon / 6) P$ and hence cuts through one of the dark clouds within $(1+2 \varepsilon / 3) \mathbb{B}^{d-1}$. Therefore $L$ intersects a ball of the corresponding dark cloud which is completely contained in $((1+$ $\left.\varepsilon) \mathbb{B}^{d-1}\right) \backslash \mathbb{B}^{d-1}$.

Step 3. Now we construct a suitable finite packing of spherical caps on $\mathbb{S}^{d-1}$.

Let $\alpha, \beta \in(0,1)$ with $\alpha<\beta$, and let $S=\left\{x \in \mathbb{S}^{d-1}: \beta-\alpha \leq x_{d} \leq \beta\right\}$. The radial projection of $S$ on the hyperplane $H=\left\{x \in \mathbb{E}^{d}: x_{d}=1\right\}$ forms an annulus $A$ in $H$, for which we apply the previous part of the construction. Let $\hat{\mathcal{C}}$ denote the set of all balls in the correponding dark clouds within $A$ and let $\hat{C}$ denote its union. Then $\hat{C}$ is met by every line in $H$ that intersects the projection of the cap $D=\left\{x \in \mathbb{S}^{d-1}: \beta \leq x_{d}\right\}$ on $H$. Let $\mathcal{C}$ be obtained from $\hat{\mathcal{C}}$ by replacing each $(d-2)$-dimensional ball by the $d$-dimensional ball with the same radius and center, and let $\mathcal{E}$ be the set of cones formed by all rays issuing from 0 that meet a single ball of $\mathcal{C}$, i.e.,

$$
\mathcal{E}=\{\operatorname{pos}(C): C \in \mathcal{C}\}
$$

Then by the assumption about the dark clouds, the cones of $\mathcal{E}$ that belong to the balls of a single dark cloud are disjoint except for 0 . Also, since $P$ is a simple polytope, each connected component of $\bigcup_{E \in \mathcal{E}}$ int $(E)$ contains at most $d-1$ cones and can be covered by a single cone issuing from 0 and spanned by a ball in order to produce a packing of cones. The intersection of these cones with $\mathbb{S}^{d-1}$ has the property to be a packing of sperical caps such that each great 2-circle that meets $D$ intersects at least one of the caps. Clearly, the packing is confined to a spherical annulus whose parameters can be adjusted as needed.

Step 4 . We now complete the construction.

Every great 2-circle on $\mathbb{S}^{d-1}$ meets one of the hyperplanes $J_{i}=\left\{x \in \mathbb{E}^{d}: x_{i}=\right.$ $1 / \sqrt{d}\} ; i=1, \ldots, d$. Now we apply Step 3 to each of the regions

$$
S_{i}=\left\{x \in \mathbb{S}^{d-1}: \frac{1}{\sqrt{d}}-\varepsilon \leq x_{i} \leq \frac{1}{\sqrt{d}}\right\} .
$$

Handling overlapping caps as in Step 3, we obtain a set of spherical caps with the required properties.

\section{A Strongly Isoradial Body}

Now we are ready to prove the main result:

Theorem 5.1. There are non-spherical strongly isoradial bodies of any dimension $d \geq 2$. 
Proof. We have already mentioned that in the plane the bodies of constant breadth are strongly isoradial and vice versa. Hence we concentrate on $d \geq 3$.

Because of Lemma 3.4 we only have to show that there exists a convex body $C$ such that $r_{1}(C)<R_{2}(C)=\cdots=R_{d}(C)$.

The idea of our construction is to choose a certain union $T$ of closed regions on the unit sphere and take their convex hull. The regions are chosen such that

(i) for any point $x$ in $T$, the antipodal point on the sphere is not in $T$, and

(ii) each great 2-circle of $\mathbb{S}^{d-1}$ meets $T$ in three points, whose convex hull contains 0.

By the first property, the diameter of the constructed convex body is strictly less than 1 while the second property guarantees that $R_{2}(C \cap F)=1$ for every plane $F \in \mathcal{L}_{2, d}$ and hence that $R_{2}(C)=R_{d}(C)$.

To construct $T$ let $\pm C_{1}, \ldots, \pm C_{m}$ be caps according to Theorem 4.1. For $i=1, \ldots, m$ let $\beta_{i} \in(0,1)$ and $u_{i} \in \mathbb{S}^{d-1}$ such that $C_{i}=\left\{x \in \mathbb{S}^{d-1}:\left\langle x, u_{i}\right\rangle \geq \beta_{i}\right\}$. Further, let $\eta$ be a sufficiently small positive number and let

$$
A_{i}=\left\{x \in \mathbb{S}^{d-1}: \beta_{i}-2 \eta \leq\left\langle x, u_{i}\right\rangle \leq \beta_{i}-\eta\right\}, \quad i=1, \ldots, m
$$

$A_{i}$ is a small spherical annulus just below $C_{i}$ and disjoint from $C_{i}$. Note that we do not produce annuli for $-C_{i}$. Since $\pm C_{1}, \ldots, \pm C_{m}$ are pairwise disjoint, we may choose $\eta$ sufficiently small, such that the union of all the $A_{i}$ 's does not contain antipodal points and such that all $A_{i}$ are disjoint from each other and from all the sets $\pm C_{i}$.

Our set $T$ is then just the union $\bigcup_{i=1}^{m} A_{i} \cup \bigcup_{i=1}^{m}\left(-C_{i}\right)$. By construction $T$ does not contain antipodal points of the sphere. Further, if $D$ is a great 2-circle, then $D$ meets some cap $C_{i}$ in some point $x$, say. Since the annulus $A_{i}$ lies below $C_{i}$ it follows that $D \cap A_{i}$ contains two points $y$ and $z$ such that $x, y, z$ lie in the same open half-circle of $D$ and $x$ lies between $y$ and $z$ on this half-circle.

Since also $-x \in-C_{i} \cap D$, we have $0 \in$ relint conv $\{-x, y, z\}$ with $-x, y, z \in T \cap D$. Hence $T$ has the desired properties (i) and (ii). Thus $\operatorname{conv}(T)$ is totally isoradial.

\section{Conclusions}

By Blaschke's selection theorem it is clear that there must exist a strongly isoradial body with maximal ratio $R_{d} / r_{d}$. Computing the maximal ratio (let alone characterizing the corresponding convex bodies) seems to be extremely hard, but even good lower or upper bounds of the maximal ratio would be interesting.

It is not too hard to see that the construction of dark orbits can be extended to general Minkowski spaces whose unit ball is strictly convex. Hence we can construct convex bodies in such spaces for which $r_{1}<R_{2}=\cdots=R_{d}$. However, it is not known whether the completion within the unit sphere can be done in general Minkowski spaces and, even if it can, in non-euclidean spaces complete bodies (of dimension at least 3) are not necessarily of constant breadth [14]. 


\section{References}

1. P.K. Agarwal, B. Aronov, and M Sharir. Line transversals of balls and smallest enclosing cylinders in three dimensions. Discrete Comput. Geom., 21:373-388, 1999.

2. K. Ball. Ellipsoids of maximal volume in convex bodies. Geom. Dedicata, 41:241-250, 1992.

3. U. Betke and M. Henk. Estimating sizes of a convex body by successive diameters and widths. Mathematika, 39(2):247-257, 1992.

4. U. Betke and M. Henk. A generalization of Steinhagen's theorem. Abh. Math. Sem. Univ. Hamburg, 63:165-176, 1993.

5. U. Betke, M. Henk, and L. Tsintsifa. Inradii of simplices. Discrete Comput. Geom., 17:365-375, 1997.

6. W. Blaschke. Kreis und Kugel. Veit, 1916.

7. H.F. Bohnenblust. Convex regions and projections in Minkowski spaces. Ann. Math., 39:301-308, 1938.

8. T. Bonnesen and W. Fenchel. Theorie der konvexen Körper. Springer-Verlag, Berlin, 1974. Translation: Theory of Convex Bodies, BCS Associates, Moscow, ID, 1987.

9. R. Brandenberg. Radii of Convex Bodies. Dissertation, Technische Universität München, 2002.

10. R. Brandenberg. Radii of regular polytopes. Accepted for publication in Discrete Comput. Geom.

11. R. Brandenberg and D. Larman. Dark clouds on spheres and totally non-spherical bodies of constant breadth. Beiträge Algebra Geom., 44:531-538, 2003.

12. R. Brandenberg and T. Theobald. Algebraic methods for computing smallest enclosing and circumscribing cylinders of simplices. Appl. Algebra Engrg. Comm. Comput., 14(6):439-460, 2004.

13. A. Brieden, P. Gritzmann, R. Kannan, V. Klee, L. Lovász, and M. Simonovits. Deterministic and randomized polynomial-time approximation of radii. Mathematika, 48:63-105, 2001.

14. G.D. Chakerian and H. Groemer. Convex bodies of constant width. In P.M. Gruber and J. Wills, editors, Convexity and Its Applications, pages 49-96. Birkhäuser, Basel, 1983.

15. M.A. Hernández Cifre and S. Segura Gomis. The missing boundaries of the Santalo diagrams for the cases $(d, \omega, R)$ and $(\omega, R, r)$. Discrete Comput. Geom., 23:381-388, 2000.

16. M.A. Hernández Cifre, J.A. Pastor, G. Salinas Martínez, and S. Segura Gomis. Complete systems of inequalities for centrally symmetric convex sets in the $n$-dimensional space. Arch. Inequal. Appl., 1:155167, 2003

17. M.A. Hernández Cifre, G. Salinas, and S. Segura Gomis. Complete systems of inequalities. J. Inequal. Pure Appl. Math, 2(1), Paper No. 10, 12 pp., 2001. Online journal; http://jipam.vu.edu.au/.

18. L. Danzer, B. Grünbaum, and V. Klee. Helly's theorem and its relatives. In V. Klee, editor, Convexity, Proc. Symp. Pure Math., Vol. 13, pages 101-180. American Mathematical Society, Providence, RI, 1963.

19. H.G. Eggleston. Convexity. Number 47 in Cambridge Tracts in Mathematics and Mathematical Physics. Cambridge University Press, Cambridge, 1958.

20. H.G. Eggleston. Notes on Minkowski geometry. J. London Math. Soc., 33:76-81, Cambridge, 1958.

21. H.G. Eggleston. Minimal universal covers in $E^{n}$. Israel J. Math., 16:149-155, 1963.

22. H. Everett, I. Stojmenovic, P. Valtr, and S. Whitesides. The largest $k$-ball in a $d$-dimensional box. Comput. Geom., 11:59-67, 1998.

23. W.J. Firey. Convex bodies of constant outer p-measure. Mathematika, 17:21-27, 1970.

24. P. Gritzmann and V. Klee. Inner and outer $j$-radii of convex bodies in finite-dimensional normed spaces. Discrete Comput. Geom., 7:255-280, 1992.

25. P. Gritzmann and V. Klee. Computational complexity of inner and outer $j$-radii of polytopes in finitedimensional normed spaces. Math. Program., 59:163-213, 1993.

26. E. Heil and H. Martini. Special convex bodies. In P.M. Gruber and J. Wills, editors, Handbook of Convex Geometry, pages 347-385. North-Holland, Amsterdam, 1993.

27. M. Henk. Ungleichungen für sukzessive Minima und verallgemeinerte In- und Umkugelradien. Dissertation, Universität-GH Siegen, 1991.

28. M. Henk. A generalization of Jung's theorem. Geom. Dedicata, 42:235-240, 1992.

29. D. Hilbert and S. Cohn-Vossen. Anschauliche Geometrie. Springer-Verlag, Berlin, 1932. Translation: Geometry and the Imagination, Chelsea, New York, 1952.

30. R. Howard. Convex bodies of constant width and constant brightness. Preprint, 2003.

31. H.W.E. Jung. Über die kleinste Kugel, die eine räumliche Figur einschließt. J. Reine Angew. Math., 123:241-257, 1901. 
32. V. Klee. Is a body spherical if its HA-measurements are constant? Amer. Math. Monthly, 76:539-542, 1969.

33. K. Leichtweiss. Zwei Extremalprobleme der Minkowski-Geometrie. Math, 62:37-49, 1955.

34. G.Y. Perel'man. $k$-Radii of a convex body. Sibinsk. Mat. Zh., 28:185-186, 1987.

35. L.A. Santaló. Sobre los sistemas completos de desigualdades entre tres elementos de una figura convexa plana. Math. Notae, 17:82-104, 1961.

36. P.R. Scott. A family of inequalities for convex sets. Bull. Austral. Math. Soc., 20:237-245, 1979.

37. P.R. Scott. Sets of constant width and inequalities. Q. J. Math., 32:345-348, 1981.

38. P.R. Scott. An extension of Blaschke's theorem in the plane. Elem. Math., 46:102-106, 1991.

39. P. Steinhagen. Über die größte Kugel in einer konvexen Punktmenge. Abh. Math. Sem. Univ. Hamburg, $1: 15-26,1921$

40. B. Weissbach. Über die senkrechten Projektionen regulärer Simplexe. Beiträge Algebra Geom., 15:35-41, 1983.

Received August 5, 2003, and in revised form March 4, 2004. Online publication August 30, 2004. 